\title{
Cartographie de l'aléa retrait-gonflement et plans de prévention des risques
}

\section{VINCENT, E. PLAT,}

S. LE ROY

BRGM

Service Aménagement et risques naturels/Risques mouvement de terrain 117, avenue de Luminy, BP 167, 13276 Marseille m.vincent@brgm.ti e.plat@brgm.fr; s.leroy@brgm.f
Depuis 1989, les mouvements différentiels de sols liés au phénomène de retrait-gonflement des argiles ont engendré en France de très nombreux sỉnistres, principalement sur les maisons individuelles. Pourtant, une information préventive adaptée permettrait de limiter considérablement cette sinistralité, qui se manifeste principalement à l'occasion des périodes de sécheresse. C'est pourquol le ministère en charge de I'environnement a confié au BRGM (service géologique nationalj la réalisation d'un programme pluriannuel de cartographie de l'aléa retrait-gonflement à l'échelle départementale, destiné à couvrir progressivement I'ensemble du territoire métropolitain d'ici 2010. La réalisation de ces cartes s'appuie sur la connaissance actuelle des formations argileuses superficielles, issue principalement des cartes géologiques éditées par le BRGM à l'échelle 1/50 000, formations qui sont caractérisées par leur susceptibilité au phénomène et par leur densité de sinistres ramenée à la surface d'affleurement urbanisée. Ces cartes, largement diffusées sur internet, constituent des supports utiles pour la sensibilisation des maitres d'ouvrages, dans le cadre d'une politique de prévention du risque, et servent de base à l'élaboration, par les services de l'État et pour les communes les plus affectées, de plans de prévention des risques (PPR).

Mots-clés : risques naturels, prévention, cartographie, aléa, retrait-gontlement, argile, sécheresse.

Since 1989 differential ground movements due to swelling and shrinkage of clayey soil have damaged a great deal of light buildings. Nevertheless preventive information campaigns could considerably imit the number of disasters which occur during drought period. That is why the environment department have asked the BRGM [French geological survey] to map the swelling and shrinkage hazard at a regional scale (about $5000 \mathrm{~km}^{2}$ ) in order to cover France entirely from present day until 2010. These maps are based on the knowledge of superticial clayey soil derived from geological maps realised by BRGM at the scale of 1:50,000. These clayey soils are characterized by their swelling and shrinkage potential and the number of disasters per urbanised area which occurs on it. These maps, available on the web, should be used for prevention and to allow local authorities to elaborate land management plans in the most concerned cities. 


\section{Introduction}

Les manifestations du phénomène de retrait-gonflement des sols argileux, ont été mises en évidence en Angleterre dès les années 1950 (Skempton, 1954) et sont désormais bien connues des géotechniciens. En France, elles ont été observées lors de la sécheresse da 1976 qui est à l'origine de nombreux désordres concernant notamment des pavillons situés en région parisienne (Philipponnat, 1978). Durant l'été 2003, plusieurs dizaines de milliers de maisons individuelles construites sur des sols argileux se sont fïssurées par suite de tassements différentiels dus au phénomène de retraitgonfiement de leur sol d'assise. conduisant pius d'une commune française sur cinc à introdulire une demande de reconnaissance de l'état de catastrophe naturelle (Vncent, 2006). Ces sinistres constiment désormais en France Je deuxième poste d'indemnisation au titre des catastrophes naturelles alors que de nombreux désordres pourrafent être évités moyennant le respect de quelques règles préventives de construction. C'est pourquoi le ministère en charge de l'envirannement a confié au BRGM (service géologique national) un programme de cartographie de l'aléa retrait-gonflement pour localiser les zones dans lesquelles la probabilité d'occurrence du phénomène est élevée et élaborer, dans les communes les plus touchées, des plans de prévention des risques (PPR) afin d'y imposer des règles constructives préventives. L'objectif de cette démarche est, d’une part, d'inciter les maîtres d'ouvages et les constructeurs de maisons individuelles à prendre cet aléa en compte dès la conception des projets et, d'autre part, d’informer un public le plus large possible quant à l'in térêt d'adopter de telles mesures préventives.

La notion d'aléa se définit par la manifestation d'un phénomène naturel (débordements de rivières, glissements de terrains, séismes, avalanches, cyclones, éruptions volcanigues, etc.), caractérisée par sa probabilité d'occurrence et son intensité, en un lieu donné. Une carte d'aléa résulte de la combinaison d'un modèle (déterministe ou issu d'analyses multicritères) et d'un système d'information géographique (SIG) elt permet d'intégrer de très nombreux paramètres de nature hétérogène, qu'ils soient ponctuels ou surfaciques létudes géotechniques, cartes de sol, modèle nunérique de terrain, etc.J. Ce type de carte a été étabil pour de très nombreux risques naturels (inondation, cavités souterraines, mouvements de terrain, séismes, etc.).

Le présent article a pour but de présenter la méthodologie retenue pour la cartographie de l'aléa retraitgonflement et les principes de transcription de ces cartes d'aléa en propositions de zonages réglementaires pour l'élaboration de plans de prévention des risques.

\section{2}

\section{Les paramètres de la susceptibilité au retrait-gonflement des argiles}

En climat tempéré, oủ les sols de surface sont souvent proches de lewrétat de saturation et ont un pouvoir de gonflement limité, ce sont surtout les périodes de sécheresse qui sont à l'origine des mouvements de terrain les plus intenses occasionnés par le phénomène de retrait-gonflement des argiles : la dessicca tion des sols argileux se traduit parfois par l'apparition de fissures de retrait en surface mais surtout par des tassements verticaux, peu visibles mais qui sont à l'origine des désordres sur le bâti. En effet, ces mouvements verticaux du sol sont rarement uniformes car leur amplitude est très variable en fonction des hétérogénéités locales concernant tant la nature du sol que son degré de dessiccation. En période de sécheresse, il apparaît ainsi des gradients importants de teneur en eau et de pression interstitielle entre des zones imperméabilisées en surface (du fait notamment de la présence d'un bâtiment) el d'aulres directement exposées à l'évaporation ou à une dessiccation encore plus profonde liée à la succion des racines d'arbres ou à un drainage par exemple. Ces gradients se traduisent par des tassements différentiels đu sol gui peuvent s'avérer très dommageables pour les constuructions lorsque celles-cl sont fondées de manière trop superficielle ou sont insuffisamment rigides pour résister à de telles solhicitations (Mouroux et al., 1988; CEBTP, 1991).

\section{2,1}

\section{La géologie : principal facteur de prédisposition}

La nature géologique des terrains affleurant ou subaffleurant constitue le principal facteur de prédisposition du phénomène. Cecỉ est clairement illustré par la carte montrant la répartition géographique, à l'échelle nationale, des communes ayant bénéficié d'au moins un arrêté de reconnaissance de l'état de catastrophe naturelle au cours des quinze dernières années (Fig. 1). Cette carte montre que la quasi-totalité du territoire métropolitain est concernée par ce phénomène qui a touché plus d'une commune française sur cinq, mais ses manifestations sont indéniablement concentrées dans certains secteurs géographiques (département du Nord, région Ile-de-France, sud du Bassin parisien, Gironde, Dordogne, région toulousaine, plaine de la Limagne. Bouches-du-Fhône, etc.) caractérisés par la présence de quelques formations géologiques argileuses (argile des Flandres, argile verte de Ronainville. mames bleues d'Argenteuil, marnes et sables de l'OrIéanais, marnes sableuses du Cénomanien, molasses du Sud-Ouest, argiles vertes à smectites de Mormoiron, etc.)

Cette corrélation spatiale entre la sinistralité observée et la nature des formations géologiques afileurantes est marufeste lorsqu'on compare la carte des communes concernées avec une carte géologique même très simplifiée (FIg. 1). En particulier, les régions de socle sont très largement épargrées par le phénomène même si localement des sinistres peuvent se produỉre du fait de la présence d'altérites ou de placages sédimentaires résiduels. A l'échelle locale de la parcelle construite, les études géotechniques réalisées dans le cadre d'expertise post-sinistres confirment largement cette analyse et indiquent la présence de matériaux argileux ou marneux dans le sol d'assise, mène si ces matériaux ne sont pas toujours jdentifiés sur les cartes géologiques disponibles car provenant de formations superficielles souvent remaniées et pariois mal cartographiées. 
a)

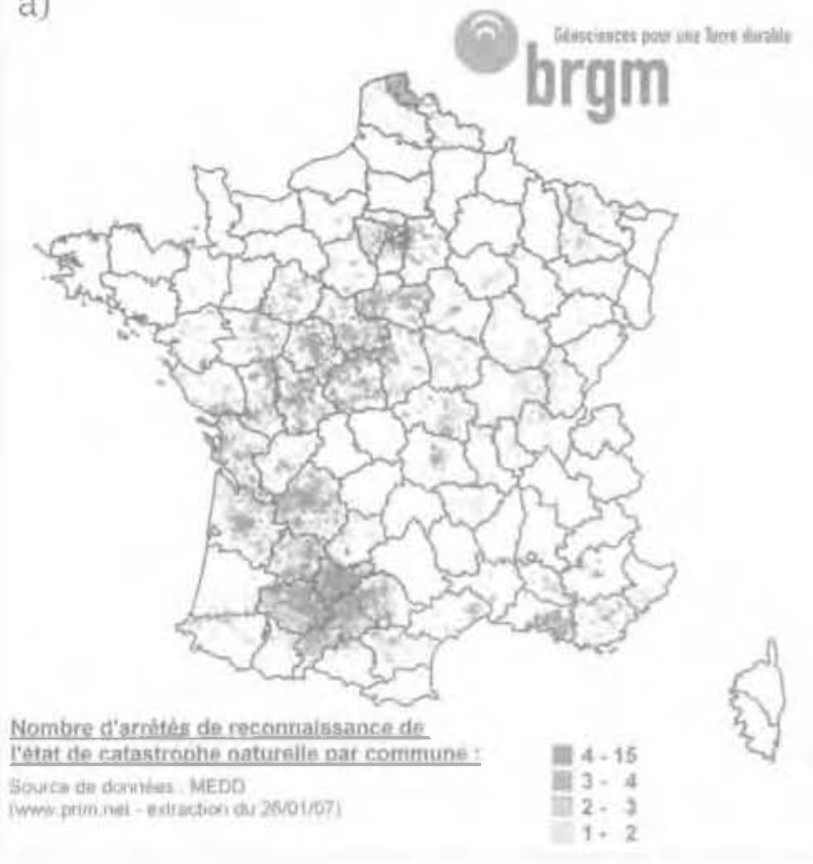

b)

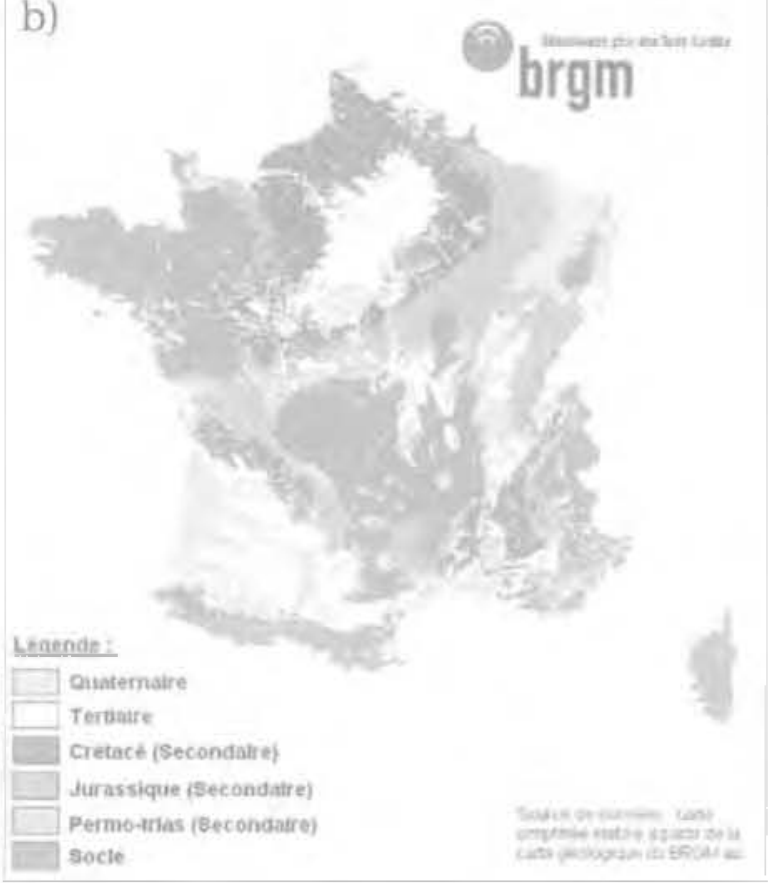

FIG. 1 Répartition des commumes françaises reconnues au moins une fois en état de catastrophe naturelle sécheresse (a) et présentation simplifiée de la carte géologique de France éditée à l'échelle $1 / 1000000$ par le BRGM (b).

Distribution of citys recogmized at least one time in natural disaster state due to drought in France (a] and sirnolified geological map of France at 1:1,000,000 edited by BRGM (b).

\section{2}

\section{Les paramètres caractéristiques de la susceptibilité des sols au retrait-gonflement}

Si à l'échelie nationale la manifestation du phénomène de retrait-gonflement est clairement reliée à la nature des formations géologiques affleurantes, ce qui a d'ailleurs conduit à l'élaboration de cartes de susceptibilité à l'échelle nationale sur la base de la carte géologique de France éditée par le BRGM à 1/1 000000 (Toulemont et al., 1994; Chassagneux et al., 1998), la réalité est localement plus complexe. Les sols en place, dans les 2 à 5 premiers mètres de profondeur, qui cor respondent à la tranche soumise aux tassements différentiels par retrait-gonflement, sont par nature hétérogènes. Il convient donc de déterminer des paramètres physiques permettant de caractériser la susceptibilité de ces sols vis-à-vis du phénomène de retrait-gonflement. La combinaison de ces paramètres, affectée éventuellement de poids variables, permel ensuite de hiérarchiser la susceptibilité globale de chacune des formations géologiques argileuses sur une base statistique qui tienne compte de cette variabilité locale.

\section{Lanat \\ Lithologie}

La nature lithologique du sol, et notamment la proportion d"élements fins argileux (de granulométrie inférieure à $2 \mathrm{\mu m}$ ) est classiquement reconnue comme un critere déterminant de l'aptitude d'un sol au retraitgonflement (Meisina et al., 1998). Il permet de distinguer les terrains essentiellement argileux, de ceux où l'argile est minoritaire. Ce critère intègre donc l'hétérogénéité des formations. En particulier, une formation de type alluvial, souvent à dominante sablo-graveleuse mais présentant des lentilles argileuses discontinues, peut être localement à l'origine de sinistres et ceci d'autant plus que ces formations alluviales ont souvent été le siège d'une urbanisation récente intensive. Il apparaît en effet que ces configurations de fortes variations lithologiques, tant horizontales que verticales, sont particulièrement dangereuses pour la stabilité des constructions implantées sans reconnaissance géotechnique préalable. La présence de lentilles ou de poches d'argile (en contexte karstique sur plateau calcaire par exemple), même de faible extension géographique, suffit de fait à occasionner des tassements différentiels préjudiciables à l'échelle d’une parcelle construite. Ceci est évidemment accentué lorsque les fondations s'appuient sur des matériaux de nature variable : ainsì une maison fondée d'un côté sur un niveau calcaire et de l'autre sur des matériaux argileux même très peı sensibles au phénomène de retraitgonflement, subira immanquablement des tassements différentiels gu'il est indispensable d'identifier dès la conception du projet.

Par ailleurs, il semble que la présence de niveaux perméables intercalés entre des temes argileux favorise les varialions hydriques saisonnières au sein de ces derniers, y compris en profondeur, ce qui est de nature à accentuer les mouvements périodiques du sol en surface. En effet, en période sèche, les circulations temporaires d'eau dans les niveaux perméables se tarissent, supprimant toute réhumidification des horizons argileux sus-jacents par remontée capillaire, ce qui accélère la dessiccation de ces derniers. Ceci explique en partie la forte sinistralité de certaines formations hétérogènes telles que les marnes et sables de l'Orléanais en région Centre, ou les argiles vertes à smectites de Mormoiron dans le Vaucluse. 
Au-delà de I'hétérogéréité intrinsèque des formations géologiques, la géométrie des termes argileux au sein de la formation entre aussi en ligne de compte, puisque les formations argileuses peu épaisses présentent un potentiel de retrait ou de gonflement moindre que les formations puissantes.

\section{9 \\ Minéralogie}

Les minéraux argileux les plus sujets au rctrait-gonflement se caractérisent par de fables liaisons internes entre feuillets et une grande surface spécifique qui leur permet d'adsorber et de relàcher de grandes quantités d'eau, ce qui se traduit par d'importantes variations de volume en cas de modification de leur teneur en eau. Les sols argileux seront donc d'autant plus affectés que leur fraction fine est riche en certains minéraux argileux, principalement de la famille des smectites (montmorillonite, beidelite, etc.), ainsi cue certains interstratifiés de type illite-smectites. La composition minéralogique de la phase argileuse est donc également un critère déterminant de la susceptibilité d"un sol au retrait-gonflement.

Le pourcentage de minéraux gonflants (à savoir smectites et interstratifiés avec présence de smectites) dans la phase argileuse est un paramètre éminemment variable, mais qui dépend néanmoins étroitement des conditions paléogéographiques de dépôt et d'évolution pédogénétique de la formation (Colas et al, 2005). Cette composition pourra donc être approchée par une analyse géologique prenant en compte d'une part les conditions de mise en place et l'environnement de dépót de la formation géologique, et d'autre part l'histoire (tectonique mais aussi climatique) subie par cette formation. Elle devra cependant être validée par une analyse quantitative fondée sur les résultats d'essais de diffractométrie aux rayons $X$ sur un nombre d"échantillons suffisamment grand pour que les conclusions tirées soient statistiquement représentatives, ce qui n'est pas toujours aisé.

L'exploitation de données spatiales obtenues avec des techniques d'imagerie hyperspectrale a aussi été envisagée pour mettre en évidence la prêsence de sols gonflants et en cartographier les contours : les images sont d'abord analysées par photo-interprétation ce quí permet des compositions colorées. Cette méthode a permis notamment de distinguer des massifs de smectite pure de zones contenant des mélanges de minéraux argileux dans le Colorado, aux Etats-Unis (Chabrillat et al., 2002]. Toutefois, Launeau ef al. [2002) insistent sur le fait que les images doivent necessairement être corrélées à des mesures in situ et que la cartographie des zones végétalisées aide largement l’interprétation de ces images. Chabrillat et al. (2002) rappellent de surcroît que la réussite d'une telle cartographie est conditionnée par l'exposition du site et sa variabilité minéralogique. Globalement cependant, et dans l'état actuel des connaissances, les conditions d'utilisation d'une telle approche semblent très contraignantes et limitées au seul cas des zones rurales non végétalisées.

\section{ogas}

\section{Comportement géotechnique}

Différents essais de laboratoire classiquement utilisés en géotechnique permettent d'approcher la sus- ceptibilité d'un matériau vis-à-vis du phênomène de retrait-gonflement mais aucur essai ne permet à lui seul de la caractériser de manière univoque car celle-ci dépend à la fois de la granulométrie du matériau, de la composition de sa phase argileuse, de sa texture, de son état de compacité, de sa teneur en eau, de son comportement mécanique, etc.

De nombreux auteurs ont néanmoins établi des corrélations empiriques entre la susceptibilité d'un sol au retrait-gonflement et certaines de ses caractéristiques physiques, hydriques et mécaniques : teneur en eau initiale, densité des grains solides, limites d'Atterberg. pourcentage des particules argilleuses, surtace spécifique, etc. (Vhcent et al, 2006). En particulter, Meisina et al. (1998) ont réalisé une étude comparative de différents essais de laboratoire susceptibles d'ètre utilisés pour caractériser le potentiel de retrait-gonflement d'un matériau, les critères examinés étant notamment la fraction du sol examiné, la qualité du prélèvement nécessaire (échantìllons intacts ou remaniés), le délai d'obtention du résultat, la simplicité de réalisation, le cout et la corrélation entre les résultats de l'essai et be comportement du sol vis-à-vis du retrait-gonflement (nulle, indicative, qualitative, quantitative ou complète). Les essais retenus comme ayant le meilleur rapport efficacité/cot̂t dans le cadre de cette étude sont les suivants : essai au bleu de méthylène, limites d'Atterberg, essai de retrait linéaire, essai de gonflement à l'cedomètre et mesure de succion au papier filtre.

L'essai au bleu de méthylêne, méthode relativement globalisante qui intègre à la fois la guantité d'élêments fins et leur capacitế d'échange, est souvent jugé comme étant le plus adapté pour une caractérisation des propriétés intrinsèques de retrait-gonflement d'un sol. Les autres essais jugés intéressants dans ce cadre sont notamment les mesures de retrait linéaire ; cellesci constituent un indicateur de l'importance du retrait volumicue possible d'un sol lors de son assèchement, ainsi que l’indice de plasticité, calculé à partir des limites d'Atterberg qui mettent en évidence l'influence de la teneur en eau sur la consistance du matériau fin.

11 importe en effet de préciser que la nature des essais géotechnicues exploitables pour évaluer la susceptibilité d'un matériau au retrait-gonflement dépend étroitement du contexte de l'étude. Dans le cadre d'une expertise géotechnique avant construction ou après sinistra, he géotechnicien s'attachere non seulement à caractériser les propriétés du sol en cas de modification de son état hydrique ou du chargement appliqué, mais s'intéressera aussi à son état au moment de l’étude. La détermination de son indice de consistance, de sa pression de gonflement ou de son potentiel de retrait, qui dépendent étroitement de sa teneur en eau naturelle, seront donc des éléments importants pour l'appréciation du comportement du sol. En revanche, dans le cadre de l'établissement d'une carte de susceptibilité au refrait-gonflement, seules les propriétés intrinsèques du sol, indépendamment de son êtat hydrigue au moment de l'étude, peuvent être prises en compte, ce qui explique l'intêrêt d'essais tels que la valeur au bleu de méthylène.

\section{Les autres paramètres de la susceptibilité}

Outre les paramètres caractérisant la nature du sol, d’autres éléments influent sur la susceptibilité d’un site 
au retrait-gonflement. En particulier, l'occupation du sol et notamment la présence de végétation arborée mais aussi de réseaux enterrés où d'éléments d'imperméabilisation discontinus à la surface du sol sont autant de facteurs de prédisposition à l'apparition de tassements différentiels en présence de sol argileux sujets au phénomène. Ces différents facteurs de prédisposition sont cependant trop ponctuels pour pouvoir être cartographiés à une échelle compalible avec la mise en œuvre d'une politique de prévention nationale.

Le contexte hydrogéologique, du moins pour ce qui concerne les nappes les plus superficielles (alluviales en particulier) et notamment les nappes perchées non pérennes, est également un facteur qui influe de maniêre directe la prédisposition de certains sites. Néanmoìns, les données concernant l'évolution piézométrique de telles nappes, souvent d'extension très localisées et sans véritable enjeu en matière de ressources en eau, est généralement très mal connue et très diffìcile à prendre en compte dans le cadre de l'établissement d'une carte de susceptibilité.

Enfin, les caractéristiques géomorphologiques d'un site influent également : un terrair en perte, exposé au Sud, sera davantage soumis à dessiccation en période estivale. Il apparaît cependant que ce critère n'est pas totalement pertinent à prendre en compte dans la réalisation d'une carte de susceptibilité (Norie et al., 2001) dans la mesure où les sinistres qui surviennent dans les zones de pente s'expliquent avant tout par la nature lithologique des matériaux mais aussi souvent par des défauts de réalisation et de conception de londations (avec fréquemment des ancrages de fondation hétérogènes, généralement trop superficiel côté aval).

\section{4}

\section{Les facteurs de déclenchement}

Pour passer d'une carte de susceptibilité à une véritable carte d'aléa affichant la probabilité d'occurrence du phénomène, il est indispensable de prendre en considération les facteurs de déclenchement. Parmi ceux-ci, les éléments climatiques sont manifestement prépondérants comme le montre la répartition temporelle de la sinistralité observée en France depuis 1989 et qui se concentre nettement au cours des périodes de sécheresse : 1989-92 (caractérisée par un déficit de pluies efficaces se prolongeant sur plusleurs années successives), 1997-98 et l'été 2003 (caractérisé surtout par de fortes températures estivales). On constate clairement que la plupart des sinistres se déclenche après des périodes soit de sécheresse intense caractérisée par une forte évapotranspiration, soit de déficit pluviométrique cumulé sur plusieurs années, les deux situations aboutissant à un approfondissement anormal du front de dessiccation dans le sol.

Les mécanismes permettant de relier ces conditions météorologiques exceptionnelles au déclenchement des sinistres dépendent cependant de multiples facteurs locaux tels que l'exposition de la parcelle, la nature du sol (notamment ses propriétés de rétention d'eau) et surtout son état de surface, généralement anthropisé (végétation, terrasses imperméabilisées, drainages, etc.), de telle sorte qu'ils sont très difficiles à quantìfier dans le détail et font d'ailleurs l'objet de plusieurs programmes de recherche. ll est donc encore prématuré, dans l'état actuel des connaissances, de prévoir la probabilité d'occurrence de futurs sinistres en tenant compte à la fois de l'évolution climatique temporelle et de la répartition spatiale des sols et du bâtí.

L'analyse d'un grand nombre de sinistres survenus depuis 1989 (CEBTP, 1991; Vincent et al., 2006) confirme de toute façon clairement que les facteurs d'origine climatique ne sont pas seuls en cause dans le déclenchement du phénomène mais que des élements, de nature très localisée, liés d'une part à la croissance des arbres et d'autre part à des facteurs anthropiques (terrassements, pompages, drainage, zones de chauffage, fuites de canalisations enterrées, etc.) prennent souvent une part prépondérante pour expliquer le déclenchement de nombreux sinistres, tout en étant trop ponctuels pour être pris en compte dans l'établis sement d'une carte d'aléa.

A défaut de pouvoir établir la probabilité d'occurrence du phénomène sur une base déterministe s'appuyant sur l'influence respective et quantifiée des facteurs de prédisposition et de déclenchement, une altemative possible consiste à évaluer cette occurrence en se basant sur l'observation statistique des phénomènes observés dans le passé. Une des voies explorées dans ce sens repose sur l'analyse des déformations du sol évaluées par imagerie spatiale selon la technique de I'interférométrie radar (par comparaison entre deux vues prises au même endroit à des dates différentes). Cette méthode a notamment été mise en ceuvre pour étudier les déformations verticales du sol dans l’Est parisien (Ben Hassen, 2005) au cours des récentes périodes de sécheresse, mais n'a jusqu'à présent pas permis de mettre en évidence de corrélation nette entre les anomalies constatées et la sinistralité observée, pourtant forte dans les zones très urbanisées bâties sur les argiles vertes de Romainville. Cette méthode a aussi été testée par plusieurs opérateurs dans la région de Garđanne (Bouches-du-Rhône), faisant apparaître de multiples divergences d'interprétation et ne permettant pas de mettre en évidence les mouvements saisonniers du sol qui étaient attendus. De même, en Lombardie (Italie), Meisina et al. (2006) mettent en évidence l'existence de mouvements de subsidence sans pouvoir établir s'il convient de les attribuer aux effets sajsonniers du phénomène de retrait-gonflement ou à la présence d'un puits de pompage au droit du site.

Des développements en cours avec l'utilisation de la technique des rélecteurs permanents permettront peut-être d'aller plus loin dans cette voie. A défaut, un moyen simple d'évaluer l'occurrence du phénomène consiste ả prendre en compte la sinistralité passée en la rapportant aux zones urbanisées, ce qui permet du moins de mettre en évidence les zones les plus touchées en l'absence de mesures de prévention. Si l'on suppose que la qualité de la construction en France n'est pas déterminée par des paramètres géographiques, cette approche donne un premfer aperçu de l'occurrence spatiale du phénomène à partir d'une quinzaine d'années d'observation et plusjeurs centaines de milliers de maisons touchées.

\section{3}

\section{Programme de cartographie de l'aléa à l'échelle départementale}

La corrélation étroite mise en évidence entre la répartition des sinistres et la nalure géologique des 
formations sub-affleurantes a amené le BRGM (Service géologique national), dès le milieu des années 1990 (Chassagneux et al., 1995), à élaborer des développements méthodologiques en vue de cartographier l'aléa retrait-gonflement des argiles, d'abord à l'échelle communale (Manosque), puis à l'échelle départementale (Alpes-de-Haute-Provence). Cette méthode, appliquée d'abord dans le département des Deux-Sèvres Vincent et al., 1998), puis dans celui de l'Essonne (Prian et al., 2000) est désormais formalisẻe [Vincent, 2003) et validée par le ministère en charge de l'environnement qui a confié au BRGM sa mise en ceuvre sur l'ensemble du tercitoire métropolitain, dens le cadre d'un programme pluriannuel qui devrait s'achever en 2010.

\section{1}

\section{Choix d'une échelle d'étude}

L'échelle de travail pour l'établissement des cartes d'aléas est naturellement fonction de la précision souhaitée, des données disponibles et des financements attendus. Les premières approches mises cuvte en France l'ont été à une échelle nationale (Toulemont et al., 1994; Chassagneux et al., 1998), sur la base de la carte géologique de la France au 1/1000 000, éditée par le BRGM. Cette approche est utile pour identifier les secteur's géographiques les plus touchés à l'échelle nationale mais s'avère bien sûr très insuffisante pour développer la prévention du phénomène en matière d'aménagement. Pour cela, il est en effet nécessaire de disposer de documents cartographiques suffisamment précis pour pouvoir déterminer avec un maximum de fiabilité le niveau d'aléa d'une parcelle donnée.

Les premières approches menées par le BRGM à l'échelle communale, sur la ville de Manosque et en collaboration avec le bureau d'étude Sol-Concept (Chassagneux et al, 1995), sont plus satisfaisantes pour un tel usage. De telles démarches ont été menées par d'autres organismes notamment sur une partie de l'agglomération montpelléraine (Combes, 1993) et plus récemment sur la commune de Toulouse (GIPEA, 2006). A cette échelle cependant, il est totalement exclu de se limiter aux seules données géologiques et géotechniques publiées, à moins de disposer de bases de données géoréférencées particulièrement détaillées, voire de modèles géotechniques en Irois dimensions. Une telle approche est en tout étal de cause couteuse et peut difficilement être mise en couve en dehors de quelques agglomérations urbaines où l'on dispose d'un grand nombre d'investigations géologiques.

Afin de répondre aux attentes des pouvoirs publics qui souhaitent développer, dans des dêlais raisonnables et à moindre coût, sur l'ensemble du terTitoire national, une politique d'affichage et de prevention du risque de retrait-gonflement, il a donc été nécessaire de mettre en cuvre une méthodologie adaptée permettant de réaliser de manière relativement rapide et homagène des cartes d'aléa à partir des seules données existantes. Pour cela, l'échelle départementale s'est révélée la plus adaptée et la donnée de base principalement utilisée est celle issue des cartes géologiques éditées par le BRGM à I'échelle 1/50 000.

\section{Méthodologie mise en œuvre}

\section{Avist.}

\section{Carte synthétique des formations argileuses}

A partir des cartes géologiques éditées par le BRGM à l'échelle 1/50 000 et couvrant la totalité du département est êtablie une carte synthétique des formations affleurantes à sub-affleurantes susceptibles de présenter localement des matériaux argileux. Ceci exige un important travail de numérisation puis d'harmonisation des cartes géologiques. Ën effet, un département moyen est généralement concerné par une quinzaine voire une vingtaine de feulles, souvent levées par des auteurs différents et à des dates variables, de telle sorte que les interprétations peuvent diverger fortement entre feuilles adjacentes, particulièrement pour ce qui concerne les formations superficielles, souvent mal représentées sur les versions anciennes des cartes géologiques.

Une fois la carte géologique harmonisée è l'échelle départementale, il convient ensuite d'identifier toutes les formations à dominante argileuse ou présentant localement des termes arglleux susceptibles d'occasionner des sinistres par retrait-gonflement. Ceci suppose de procéder à certaines réinterprétations et au regroupement de formations stratigraphiquement proches et dont la lithologie est jugée suffisamment homogène pour que leur susceptibilité au retrait-gonflement puisse être considérée comme identique. Selon les départements traités, la carte départementale des formations argileuses ainsi obtenue affiche entre une quinzaine et une quarantaine de formations retenues comme potentiellement argileuses.

\section{Élaboration de la carte de susceptibilité}

Pour chacune des formations argileuses ainsi identifiées, la susceptibilité au retrait-gonflement est alors caractérisée sur la base de trois critères qui se recoupent plus ou moins et se complètent mutuellement. Pour chacun de ces critères est attribuée à la formation une note comprise entre 1 et 4 , selon l'aptitude supposée au retrait-gonflement, ceci sur la base d'une grille unique appliquée au niveau national.

Le premier critère appliqué caractérise la lithologie de la formation, à savoir la proportion et la continuité des temes argíleux qu'elle renferme. Par convention, la note maximale est attribuée à une argile ou une marne épaisse et contirue et la note minimale à une formation hétérogène, présentant des termes argileux non prédominants et discontinus, par exemple sous forme de poches ou de lentilles. Il faut noter que cette caractérisation lithologique des formations est établie sur la base de l'expertise du géologue régional et qu'elle ne peut être totalement dépourvue d'une certaine subjectivité dans son appréciation. Sà valeur relative en vue d'une hiérarchisation des formations argileuses est cependant difficilement contestable. Le barème utilisé pour distinguer les différentes classes lithologiques est présenté dans le tableau I. 
Labsau Principe d'attribution des notes de susceptibilité des formations argileuses en fonction du critère lithologique.

Method to allot sensitivity value from soil lithology:

\begin{tabular}{|c|c|c|}
\hline Type de formation & $\begin{array}{l}\text { Suscep. } \\
\text { tibilité }\end{array}$ & $\begin{array}{c}\text { Note } \\
\text { lithologigue }\end{array}$ \\
\hline $\begin{array}{l}\text { Formation non argileuse mais } \\
\text { contenant localement des } \\
\text { passées ou des poches argi- } \\
\text { leuses (ex: alluvions avec ler- } \\
\text { tiles argileuses. calcaire avec } \\
\text { poches karstiques...) }\end{array}$ & faible & 1 \\
\hline $\begin{array}{l}\text { Formation présentant un } \\
\text { lerme argileux non prédomi- } \\
\text { nant de tyoe calcaire argileux } \\
\text { cu sable argibux }\end{array}$ & moyenne & 2 \\
\hline $\begin{array}{l}\text { Formation à dominante argi- } \\
\text { leuse, présentant un terme ou } \\
\text { une passée non argileuse (ex: } \\
\text { altemance marno-calcaire ou } \\
\text { sablo-argileuse) ou tès mince } \\
\text { (moins de } 3 \mathrm{~m} \text { ) }\end{array}$ & forte & 3 \\
\hline $\begin{array}{l}\text { Fomnation essentiellement argi- } \\
\text { leuse ou marneuse, d'épaisseur } \\
\text { superieure à } 3 \text { th et cortinue }\end{array}$ & très forte & 4 \\
\hline
\end{tabular}

Le second critère retenu concerne la composition minêralogique de la phase argileuse, et plus précisément le pourcentage moyen de minéraux gonflants (smectites et interstratifiés avec présence de smectites) dans la phase argileuse. Cet élément est déterminé à partir des données bibliographiques publiées (complétées par quelques analyses ponctuelles spécifiques) en combinant une approche qualitative, basée sur l'étude des conditions paléogéographiques de dépôt et d'évolution pédogénétique, et une analyse quantitative fondée sur les résultats d'essais de diffractométrie aux rayons $\mathrm{X}$. Les bornes retenues pour cette classification minéralogique correspondent respectivement à $25 \%, 50 \%$ et $80 \%$ de minéraux gonflants (smectites et interstratifiés) dans la phase argileuse du matériau (tableau L).

TABLFad II Principe d'attribution des notes de susceptibilité des formations argíleuses en fonction du critère minéralogique.

Method to allot sensitivity value from soil mineralogy,

\begin{tabular}{c|c|c}
$\begin{array}{c}\text { \% moyen de minẻraux } \\
\text { gontlants }\end{array}$ & Susceptibilitè & $\begin{array}{c}\text { Note } \\
\text { minéralegique }\end{array}$ \\
\hline$<25 \%$ & faible & 1 \\
\hline 25 à $50 \%$ & rnovenne & 2 \\
\hline 50 à $80 \%$ & forte & 3 \\
\hline$>80 \%$ & très forte & 4 \\
\hline
\end{tabular}

Le troisième critère utilisé est basé sur une interprétation statistique de résultats d'essais de laboratoire recueillis pour chacune des formations argileu- ses identifiées (non seulement dans le cadre d'études post-sinistre, mais également au travers d'investigations menées pour des travaux d'aménagement). Cette approche suppose une collaboration active tant des bureaux d'études géotechniques soliicités que des maitres d'ouvrages publics ou privés. Pour cette interprétation, on privilégie les essais jugés les plus représentatifs du comportement vis-à-vis du retrait-gonflement, à savoir Jes valeurs de bleu (essais Vb, indiquant la capacité d'adsorption d'eauj et le retrait linéaire sur échantillons intacts (Ri). A défaut, on tient aussi compte des indices de plasticité (Ip, calculé à partir des limites d'Atterberg et indiquant l'étendue du domaine plastíque) et du coefficient de gonflement (Cg, établi à partís d'essais cedométriques). Les seuils retenus pour caractériser la potentiel de retrait-gonflement à partir de ces essais (Chassagneux et al., 1995; Mastchenko, 2001) sont présentés dans le tableau III.

La moyenne des trois notes attribuées à chaque formation pour ces trois criteres foumit la classe de susceptibilité de la formation : si cette moyenne est inférieure à 2, la susceptibilité est considérée comne faible (note égale à 1) ; si elle est comprise entre 2 et 3 , Ia susceptibilité est moyenne (note égale à 2) et elle est considérée comme forte pour une moyenne supérieure à 3 (note égale à 3 )

\subsection{9}

\section{Prise en compte de la sinistralité}

Afin d'approcher, au moins de manière qualitative, la probabilité d'occurrence du phénomène, la susceptibilité au retrait-gonflement est corrigée en intégrant également la sinistralité observée depuis 1989. Ceci nécessite de recenser le plus grand nombre possible de sinistres survenus dans le département et de les localiser avec précision, afin d'obtenir une représentation statistique réaliste des probabilités doccurrence du phénomène. Le nombre de sinistres ainsi pris en compte atteint généralement plusieurs milliers par département (jusqu'à 5250 en Haute-Garonne). Le croisement avec la carte géologiogue permet de calculer, pour chacune des formations argileuses identifiées, une densité de sinistres qui est ramenée, pour faciliter les comparaisons, à $100 \mathrm{~km}^{2}$ de surface d'affleurement réellement urbanisée. II est en effet nécessaire de tenir compte du taux d'urbanisation qui peut présenter des disparités importantes d"un point à l'autre du département et fausser ainsi l'analyse.

La densité de sinistres rapportée à $100 \mathrm{~km}^{2}$ de zone argileuse urbanisée varie très fortement, entre 205 (Eure-et-Loir) et 3354 (Tarn), avec une valeur moyenne de 1143 . Ce critère met en évidence la sinistralité particulièrement élevée de certains départements qui n’apparaissent pourtant pas comme tels lorsque d'autres critères plus globalisants sont pris en compte. Ainsi, le

TAblesul| Príncipe d'attribution des notes de susceptibilité des formations argileuses en fonction du critère géotechnique.

Method to allot sensitivity value from soil geotechnical characterization..

\begin{tabular}{|c|c|c|c|c|c|}
\hline $\begin{array}{c}\text { Valeur de bleu }\left(\mathrm{cm}^{3} / \mathrm{g}\right) \\
\text { (paramètre déterminant) }\end{array}$ & Retrait linéaire & $\begin{array}{l}\text { Coefficient } \\
\text { de gonflement }\end{array}$ & $\begin{array}{c}\text { Indice de } \\
\text { plasticite }(\%)\end{array}$ & Susceptibilité & $\begin{array}{c}\text { Note } \\
\text { géotechnique }\end{array}$ \\
\hline$<2,5$ & $<0,4$ & $<0,025$ & $<12$ & Faible & 1 \\
\hline 2,5 ̀े 6 & 0,4 a 0,65 & 0,025 aे 0,05 & 12 à 25 & Moyentie & 2 \\
\hline 6 à 8 & $0,65 \mathrm{à} 0,75$ & 0,05 à 0,09 & 25 ¿े 40 & Forte & 3 \\
\hline$>8$ & $\geq 0,75$ & $\geq 0,09$ & $\geq 40$ & Très forte & 4 \\
\hline
\end{tabular}


département des Alpes-de-Haute-Provence, faiblement urbanisé, dans lequel ont été recensés 1279 maisons sinistrées, présente une densité de 2926 sinistres pour $100 \mathrm{~km}^{2}$ de zone argileuse urbanisée, alors qu'il n'apparaît qu'à la ving tième position en terme de coût cumulé d'indemnisation, loin derrière les Yvelines ou la HauteGaronne dont les densités de sinistres sont pourtant moindres (respectivement 2542 et 1586 ).

\section{9.4}

\section{Élaboration de la carte départementale d'aléa}

La carte départementale d'aléa dérive in fine de la carte des formations arglieuses, après hiérarchisation de ces demières en fonction de leur niveau de susceptibilité et de la sinistralité quì y a été constatée. Cette combinaison de la note de susceptibilité et de la densité de sinistre par formation se fait cependant en attribuant un poids deux fois plus faible à cette dernière car celle-ci est entachée d'un certain nombre d'incertitudes quant aux causes exactes de certains des sinistres pris en considération (Fig. 2). L'échelle de validité de la carte ainsi obtenue est celle de la donnée de base utilisée, sachant que les cartes géologicues sont levées ả l'échelle du 1/25000 puis restituées au 1/50 000.

\section{État d'avancement du programme}

Après Ies développements méthodologiques initiaux et les premières applications réalisées ả la demande des assurances (Centre européen de prévention des risques pour le département des Deux-Sèves, Caisse centrale de réassurance pour celui de l'Éssonne), ces cartes d'aléa sont désormais produites dans le cadre d'un co-financement entre le Fonds national de pré vention des risques naturels mobilisé par le MEDD et la dotation de service public du BRGM. A fin novembre 2007, ces cartes d'aléa sont d'ores et déjà réalisées pour 42 départements et accessibles au public sur internet pour 35 d'entre eux (Fig. 3), après validation par la préfecture et la direction départementale de "Étuipement. Une quarantaine d'autres cartes départementales d'aléa, actuellement en cours, devraient être publiées $\mathrm{d}^{*} i c i$ fin 2007.

\section{4}

\section{Exploitation des cartes d'aléa}

Les cartes d'aléa réalisées peuvent servir à la fois comme support d'information préventive et pour l'élaboration de documents à valeur réglementaire que sont les plans de prévention des risques naturels prévisibles (PPR).
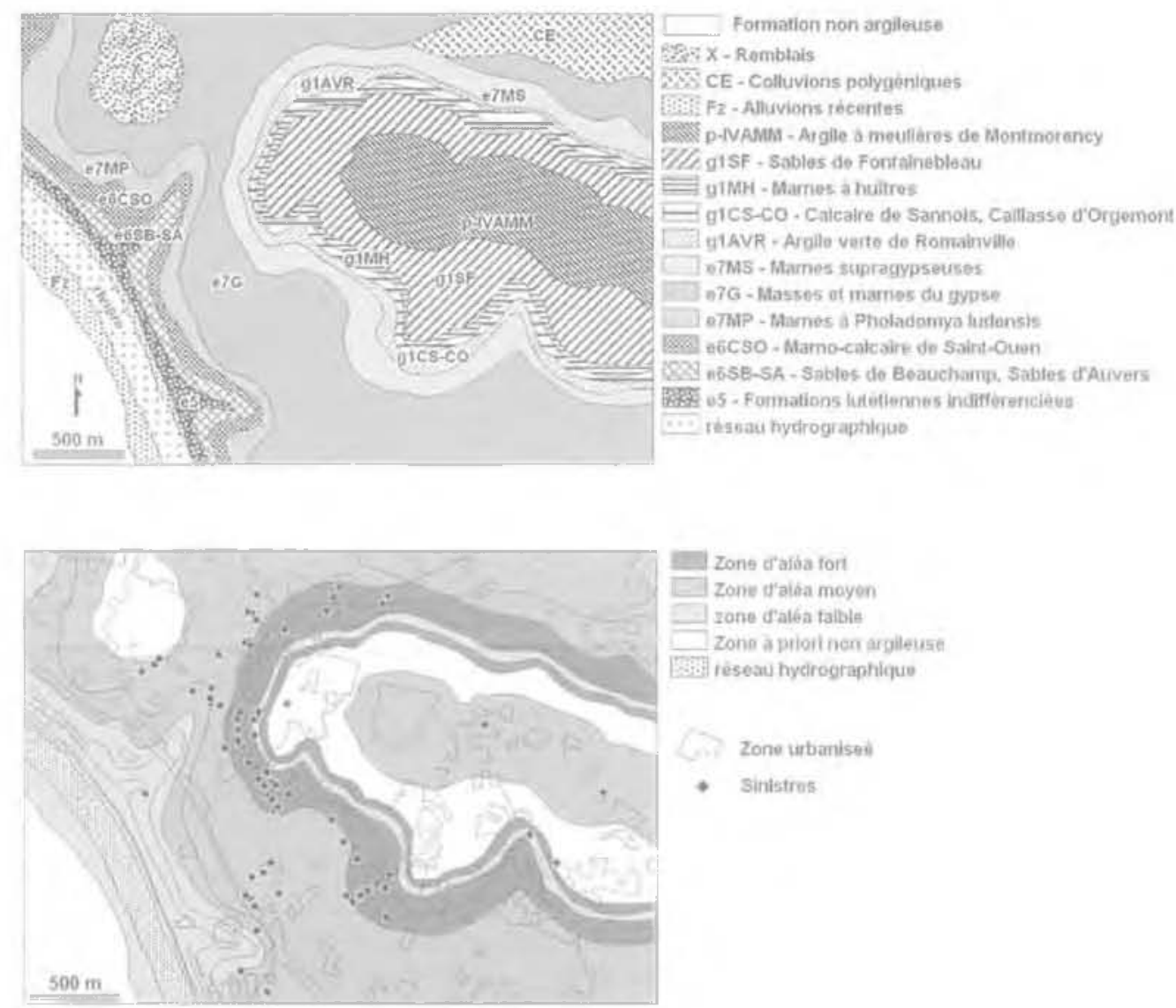

FG. Extrait de la carte synthétique des formations argileuses et de la carte d’aléa retraitgonflement du Val d'Oise (Donsimoni et al. 2004).

Extract from maps of superficial clayey soil (a) and of soil swelling and shrinkage hazard (b) (Donsimoni et al., 2004). 

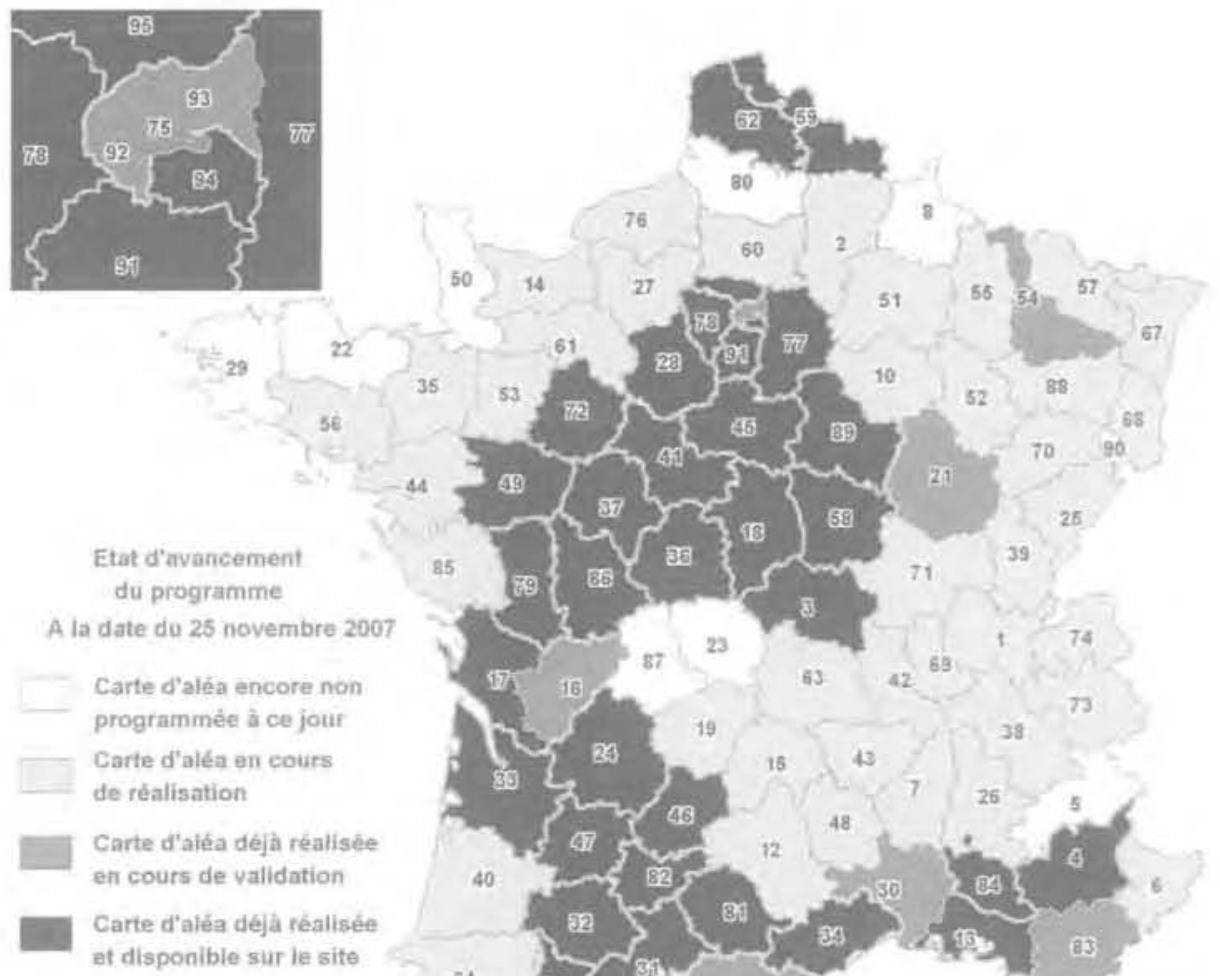

3อ

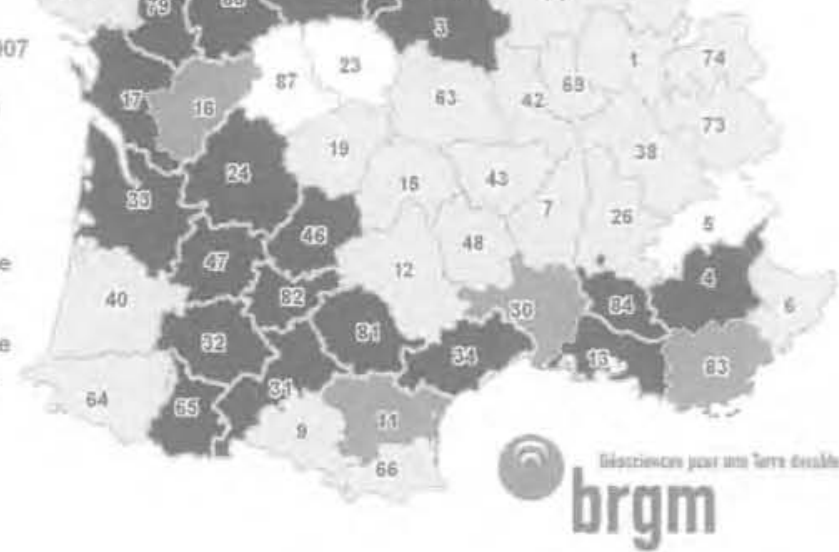

na. 3 Etat d'avancement à fin novembre 2007 du progranme de cartographie départementale de l'aléa retrait-gonflement des sols argileux. Progress report of shrinkage and swelling hazard mapping in France in November 2007.

\section{Les cartes d'aléas comme support d'information préventive}

La diminution future de la sinistralité des maisons individuelles causée par le phénomène de retrait-gonflement des sols argileux passe par une diffusion la plus large possible des mesures de prévention. C'est justement l'objectif principal de ces cartes d'aléa que d'attirer l'atiention des maîtres d'ouvrages (y compris des particuliers) et des professionnels de la construction sur la nécessité de prendre des précautions particulières lors de la construction d'une maison individuelle dans un secteur susceptible de contenir des argiles sujettes au retrait-gonflement. Dans ce domaine de nombreuses actions d'information ont été entreprises récemment, tant par les pouvoirs publics que par certains assureurs et des professionnels de la construction. C'est ainsi qu'à la demande du MEDD, le BRGM a élaboré un site internet spécifique, librement accessible à I'adresse suivante : http://wwwargiles.fr, et destiné à l'affichage des cartes d'aléa au fur et à mesure de leur publication. Ouvert au public depuis novembre 2004, ce site permet de consulter les cartes d'aléa par département ou par commune, de s'informer sur les manifestations du phénomène et la manière de les prévenir, et de télécharger Jes rapports et les cartes d'aléa déjă parus. Depuis fin février 2006, les cartes d'aléa sont disponibles sur ce site pour 35 départements, les plus concernés par le phénomène. La consultation importante de ce site (environ 50000 visiteurs par mois depuis début 2006 et 350000 durant les deux premières années de mise en service) confirme la forte attente du public vis-à-vis de ce type d'information préventive. Il est notamment très utilisé par les constructeurs de maisons individuelles et lors des transactions immobilières.

\section{2}

\section{Transposition des cartes d'aléas en zonage réglementaire}

Parallêlement, dans les secteurs les plus touchés par le phénomène, les cartes d"aléa peuvent aussi ser. vir à l'élaboration de plans de prévention des risques naturels prévisibles (PPR). L'échelle de validité des cartes départementales d'aléa retrait-gonflement est in fine celle de la donnée de base utilisée, à savoir le 1/50 000. Elles ne permettent donc pas de déterminer avec certitude la présence d'argile gonflante à l'échelle d'une parcele, surtoul si celle-ci est peu étendue. Elles suffisent néanmoins à circonscríre les zones potentiellement sujettes au phénomène de retrait-gonflement et où il convient de prendre des précautions particulières avant de construire. Établir des cartes d'alća à une échelle plus précise exigerait nécessairement de réaliser des investigations complémentaires coûteuses (avec sondages et essais en laboratoire) ; c'est pourguof, le MEDD a décidé, pour ce phénomène très particulier qu'est le retrait-gonflement qui n'entraîne pas de risque pour les vies humaines et pour lequel les 
mesures de prévention sont relativement simples et peu cotiteuses, de limiter la connaissance des zones à riscque à cette échelie du 1/50 000 .

\section{1}

\section{Méthode de transcription}

Les cartes départementales d'aléa, servent donc directement de support à l'élaboration de plans de zonage réglementaires pour la réalisation des PPR, élaborés par commune et présentés sur fond topographique extrait des cartes IGN à 1/25 000 agrandis à 1/10 000 ou sur fond cadastral (Fig. 4). Afin ce tenir compte de l’imprécision du tracé des contours dessinês à partir de documents publiés à l'échelle 1/50 000, une bande de sécurité d'une largeur de $50 \mathrm{~m}$ est intégrée pour élargir les zones d'aléa. Deux zones régtementée sont distinguées: une zone fortement exposée qui coincide avec les zones classées en aléa fort et une zone faiblement à moyennement exposée, qui régroupe les secteurs classées en aléa faible à moyen (Norie et al., 2000).

\section{Prescriptions réglementaires}

Contrairement à d'autres risçues naturels tels que les inondations, les PPR proposés ne prévoient aucune mesure d'interdiction de construire, même dans les zones considérées en aléa élevé. Les mesures préventives préconisées n’entrainent pas de surcoût important et restent donc compatibles avec la construction de maisons individuelles économiques (Exbrayat, 2001). La reconnajssance géotechnique préalable n'est pas rendue obligatoire pour les maisons individuelles (hors permis groupé). Elle est cependant vivement consellée car elle peut permetire, moyenuant un investissement relativement faible, de vérifier le contexte géologique local et de déterminer les mesures constructives les plus adaptées (voire de s'affranchir de toute contrainte particulière si elle met en évidence l'absence d'argile gonflante au droit du projet). A défaut d'une telle étude de sol, le règlement du PPR définit pour l'essentiel des mesures concernant la construction (avec notamment une profondeur minimale d'ancrage des fondations fixée à $0,80 \mathrm{~m}$ en zone fablement à moyennement

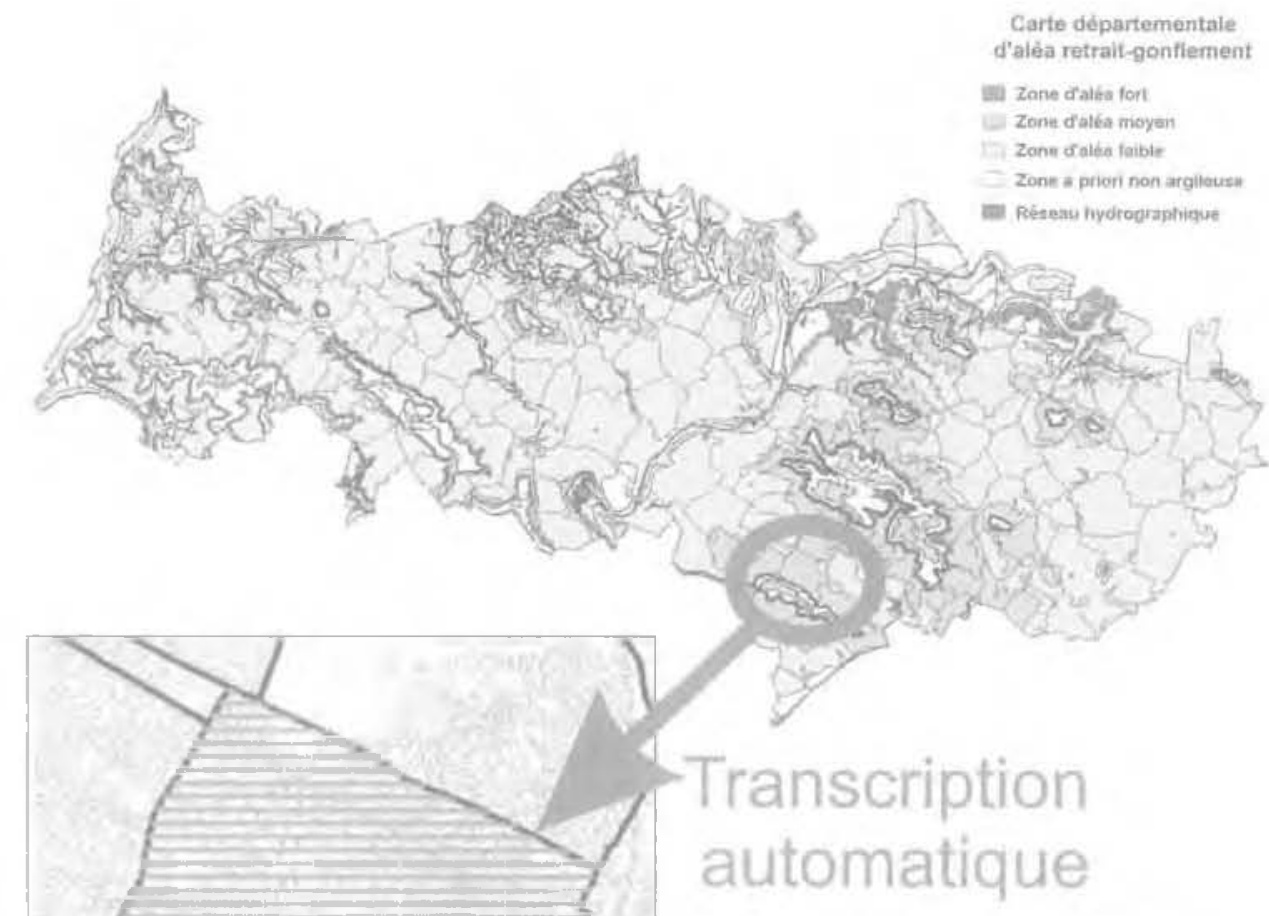

Plan de zonage réglementaire de la commune de Franconville

Zone fortement exposée (B1) Zone faiblement à moyennement exposée (B2)

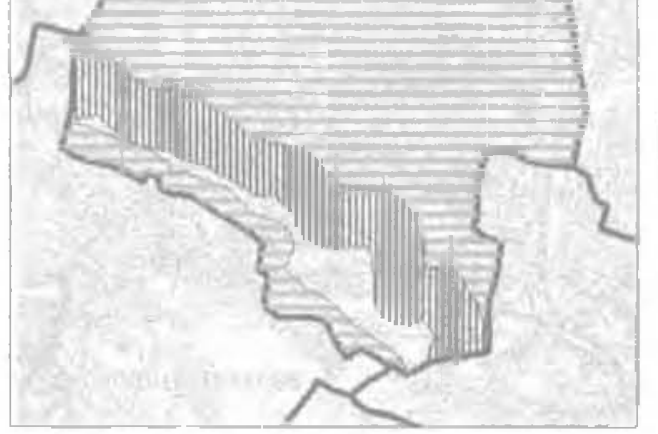

nG. Principe de transcription de la carte départementale d'aléa en propositions de zonages réglementaires pour la réalisation de PPA (Vincent et al,, 2003).

Transcription method of shrimkage and swelling hazard map in risk prevention plans (Vincent et al., 2003). 
exposée et à 1,20 $\mathrm{m}$ en zone fortement exposée) et l'environnement immédiat du projet (distance minimale à respecter pour les plantations d'arbres, dispositif pérímétrique anti-évaporation, maîtrise des eaux de ruissellement, êtanchéité des réseaux enterrés, etc.).

Les premiers PPR établis sur cette base ont été mis à l'enquête publique dans les quarante communes de Seine-Saint-Denis en novembre 2002. Plus de 1600 PPR concernant spécifiquement l'aléa retrait-gonflement des argiles ont d'ores et déjà été prescrits (Fig. 5), dont plus de 550 adoptés à fîn 2007, et d'autres pourraînt l'être très prochainement, moyennant un travail

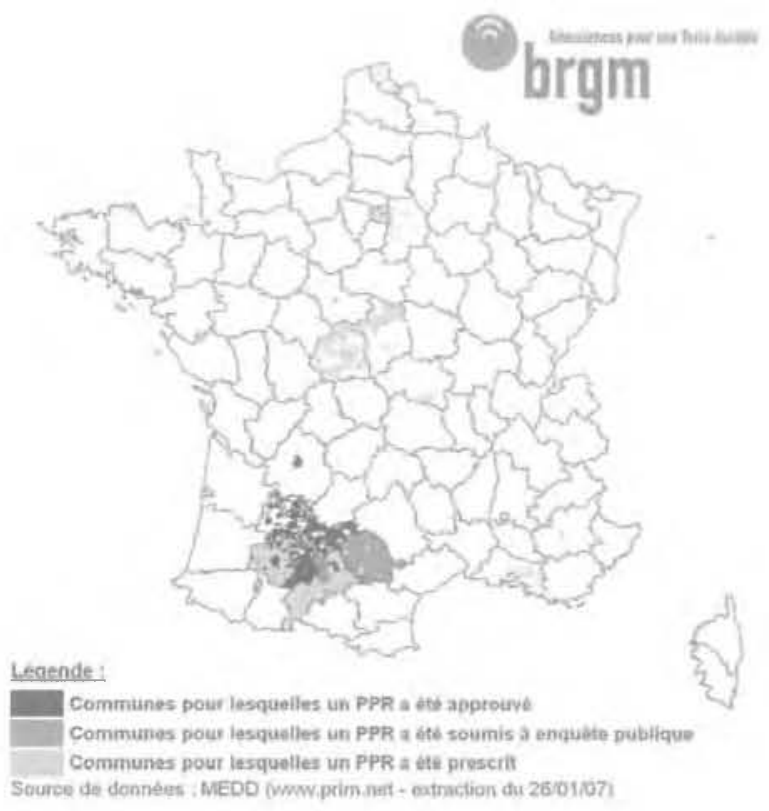

FG. 3 Etat d'avancement à fin novembre 2007 des procédures relatives aux plans de prévention des risques mouvements de terrain différentiels consécutifs à la sécheresse et à la réhydratation des sols.

Progress report of risk prevention plans procedure relative to swelling and shrinkage behaviour of clayey soil actualized in November 2007 d'adaptation du règlement afin d'ajuster certaines prescriptions concernant notamment l'impact des arbres et les rejets dans les réseaux. La mise en ceuvre de ces PPR dans les communes les plus touchées devrait permettre à l'avenir d'éviter de nombreux sinistres, traumatisants pour les propriétaires et coûteux pour la collectivité.

\section{CONCLUSION}

Si le phénomène de retrait-gonflement des sols argileux et les mesures constructives adaptées à ce type de terrain sont bien connus des géotechniciens, encore convient-il d'afficher largement le risque à I'usage des maîtres d'ouvrages et de diffuser les mesures préventives nécessaires. C'est tout l'enjeu de ce programme de cartographie départementale de l'aléa retrait-gonflement, initié par le ministère en charge de l'environnement et dont la réalisation a été confiée au BRGM afin de traiter progressivement l'ensemble du territoire métropolitain selon une méthodologie unique et à moindre coût pour la collectivité. Cette approche s'appuie sur les données géologiques disponibles afin de cartographier les contours des formations argileuses affleurantes puis évalue le niveau d'aléa de ces dernières par combinaison d'une note de susceptibilité et d'une note de sinistralité en affectant un poids moindre à cette dernière. La carte ainsi obtenue, bien qu'imprécise à l’êchelle de la parcelle, constitue une première étape indispensable pour la prise en compte du phénomène et la mise en ceuvre d'une véritable politique d'information préventive à destination des différents acteurs de la construction. Elle permet aussi, moyennant l'intégration d'une bande de sécurité, d'établir des plans de prévention des risques qui visent à circonscrire les zones potentiellement sujettes au phénomène de retrait-gonflement et où il convient de prendre des précautions particulières avant de construire. A fin novembre 2007, les 35 départements les plus touchés par le phénomène sont couverts par une carte d'aléa, mise à disposition d'un large public sur le site intemet http //wwwargiles.fr, et plus de 550 PPR ont d'ores et déjà été adoptés. 
Ben Hassen M. - Apport de l'interférométrie radar pour l'étude des faibles déformations engendrées par le comportement des matériaux argileux sous climat extrềne : application ầ la région de Lagny (1'Est parisien). Rapport de DEA présenté à l'université de Marnela-Vallee, 2005, $75 \mathrm{p}$.

CEBTP - Détermination des solutions adaptées ả la réparation des désordres des bâtiments provogués par la sécheresse, sous l'égide de l'AQC, l'APSAD I'AFAC, la CCR et la FNB. Guide pratique CEBTP, 1991, 3 fascicules.

Chabrillát 5., Goetz A.F.H., Olsen H.W. Krosley L. - Use of hyperspectra] images in the identification and mapping of expansive clay solls and the role of spatial resolution. Remote Sensing of Environment. 82 (2-3), 2002, p. $431-445$.

Chassagnemex D. Stieltjes L., Mouroux P. avec la coll. de Ducreux G.H. - Cartographie de l'aléa retrait-gonflerrent des sols (sécheresse/pluie) dans la région de Marnoscue (Alpes-ce-Haute-Provence). Echelle communale et départementale. Approche méthodologique. Rapport BPGM R 38695, 1995

Chassagneux D., Meisina $C$. vincent $M$., Mérillet F, Baudu R. - Guide synthétique pour la prise en compte de l'aléa retrait - gonflement è l'échelle. Rapport BRGM $n^{\circ}$ P40355, 1998, 33 p. 6 fig., 1 tab., 1 ann., $1 \mathrm{pl}$. h.-t.

Colas B. avec la collaboration de Le Strat P. Vincent M., Dugast D. et Lenfant S. - Cartographie de l'aléa retrait-gonflement des arciles dans le département de l'Hérault. Rapport BRGM/RP-54236FR, 2005. 123 p. 54 ill., 6 ann. 3 cartes h. - t.

Combes P. - Cartographie des zones gêologiques exposées à des risques naturels dans le département de l'Hếrault, le cas des argiles. Rapport GEDTER GIR CGE/0893-14, 1993.

Donsimoni M., Hatton C., Giraud F, avec la collaboration de Vincent M. - Cartographie de l'aléa retrait-gonflement des sols argileux dans le département du Val-d'Oise. Rapport BRGM/RP-52598FR, 2004,163 p. 8 fig., 22 tabl., 4 ann., 4 pl. h. $-\mathrm{t}$.

Exbrayat L. - Dispositions constructives de nature à prévenir et/ou supprimer les effets de la dessication/réhydratation des sols : évaluation des coûts. SOLEN Géotechnique n० ${ }^{\circ}$ G01339GT, 2001.
GIPEA avec la collaboration de 2GH Entre prise, $A D R E T$ - Risque naturel retrait gonflement des argiles. Étude spécifique pour le plan de prévention des risques natureks sécheresse applicable a Toulouse. Rapport tinal R73.06, 2006, 102 p.. 5 and

Launeau P., Sotin C., Girardeau J. - Cartographie de la péridotite de Ronda [Espagne) par télédétection hyperspectrale donnés AVIRIS. Bulletin de la Soctété geolugique de France, 2002, vol. 173; $n^{\circ}$ 8; p. $491-508$.

Mastchenko A - Sécheresse et sols argitelix. Projet industriel Alpha Sol. Ecole des Mines d'Alès, 2001, 74 p. 9 anr.

Maisina C. Chassagneux D. Leroi E. Mouroux P. - Gartographie de l'aléa retrait-gonflement des sols argileux. PToposition de méthodologie. Article ef présentatior au $8^{\circ}$ Congrès de l'Association internationale de géologie de l'ingénieur el de l'environnement, 1998.

Meisina C., Zucca E., Fossati D, Ceriani M. Allievi J. - Ground deformation monitoring by using the ameanent Scatterers Technigue: The example of the Oltrepo Pavese (Lombardia, Italy). Engineering Geology, 2006, vol, 88, p. 240-259.

Mouroux P., Maruron P., Pinte J.-C. (1988) - La construction économique sur sols gonflants. Edit. du BRGM, Mantzels et Méthodes $\pi^{\circ}$ 14, 1988

Norie A., Vincent M - Etablissement de plan de prévention des risques naturels prévisibles : mouvements différentiels de terrain liés au phénomène de retrait gonflement des sols argtleux. Approche méthodologique dans le département des Deux-Sevres. Rapport BRGM/RP50591-FR, 2000, 14 p.. 4 fig.. 4 ann

Norie A., Capdeville J.-P., Vincent M., en collaboration avec Delpont G., Schoen R., Krieg N. - Cartographie de l'aléa retrait-gonflement des argiles dans le département du Gers. Bapport BRGM RP-5125:-FR, 2001, 68 p., 23 fig., 2 pl., 8 tabl. 2 cartes h. -4 . 6 ann.

Philipponлat G. - Désordres dus à la présence de sols gonflants dans la région. parisienre. Annales de l'Institut technfque du bätiment et des travaux publics, $1978, n^{\circ} 364$, p. 1-15.

Prian J -P. Donsimoni M. Vincent $M$. avec la collaboration de Denis L., Gallas J.C. Marty F. Motteau M. - Cartographie de l'aléa retrait-gonflement des argiles dans le département de l'Essonne. Rap- port BRGM n ${ }^{\circ}$ RP-50376-FR, 2000, 269 p., 32 fig., 11 tabl., 6 ann, 3 cartes h.-t.

Skempton A.W. - A foundation failure due to clay shrinkage caused by poplar trees. Proc. instr. Giv. Engrs. Part I, 3 , $\operatorname{Jan} .1954$, p. $66-83$.

Toulemont M., Cojean R., Faccendini J.P. - Cartographie prévisionnelle des sols sensitbles à la sécheresse: in outil d'information préventive. Mappemonde, 4. 1994, p. 2-4.

Vincent M. Le Nindre Y.M. Meisina C. Chassignol A.L. - Cartographte de l'aléa retrait-gonflement des argiles dans le département des Dewx-Sevres. Papport BRGM n R $39967,1998,89$ p., 14 fig., 13 tabl, 6 ann. 2 cartes hors-texte.

Vincent M. (2003) - Retatit-gonflement des sols argileux: méthode cartographique d'evaluation de l'aléa en vue de l'établissement de PPR, ze Conférence SIRNAT, Forum cles joumées nour la prévention des risques natureks, Orléans, janv. 2003. 7 p., 5 fig.

Vincent $M$. avec ta collaboration de Imbault M. et Donsimoni M. - Établissement de plans de prévention des risgues naturels concernant les tnouvernents différentiels de terrain liés au phénomène de retrait-gonfiement des argiles dans le département du Val-de-Marne. Rapport BRGM/RP-52494-FR, 2003, 14 p., 2 fig., 1 ann., 1 CD-Rorn.

Vincent M. (2006) - Retrait-gonflement des sols argileux : un aléa géologique lié aux conditions climatiques - Gécosciences (la revue du BRGM pour une Terre durable), $n^{\circ} 3$, mars 2006, p. 50 à 55 .

Vincent M.. Bouchut j., Fleureau J.M. (LMSSMat), Masrouri F. (LAEGO). Oppenheim E. (CEBTP-Solen), Heck J.V. (CSTB), Ruaux N. (CSTB), Le Roy S., Dubus I., Surdyk N. - Eitude des mécanismes de déclenchement du phénomène de retrait-gonflement des sols argileux et de ses interactions avec le bẩi. Rapport final. BRGM/RP-54862-FR, 2006, 378 p. 308 i it. 